\title{
chę
}

\section{A emergência do campo de políticas públicas de dados abertos governamentais no Brasil}

\section{Fernando Almeida Barbalho}

Doutor em Administração pela Universidade de Brasília - UnB

\begin{abstract}
Resumo
Este artigo proporciona uma leitura das políticas públicas de produção e consumo de dados abertos, segundo a teoria de campos de ação estratégica. No Brasil, o campo de dados abertos governamentais se caracteriza como emergente, já que ainda não se estabilizou. Percebe-se que cinco grupos atuam em dinâmicas de cooperação e enfrentamento: a) Estado; b) unidades internas de governança; c) organizações da sociedade civil; d) universidades; e e) empresas. As dinâmicas do campo são influenciadas pela posse de recursos, ou capitais, de acordo com a terminologia de Pierre Bourdieu. Os capitais identificados são: a) técnico; b) social; c) simbólico; d) econômico; e e) informacional. A partir de análise qualitativa de narrativas dos sujeitos que participaram da pesquisa, o estudo apresenta uma agenda para negociação e ação, envolvendo os diversos participantes do campo. Destaca-se que essas narrativas estão associadas às funções da transparência, que se correspondem com as preocupações de governo aberto trazidas, principalmente, por agentes legitimadores internacionais. Sob o ponto de vista acadêmico, a pesquisa contribuiu ao mostrar uma forma de operacionalizar conceitos propostos por teorias dedicadas a campos de ação estratégica, sobretudo no que diz respeito ao estado de emergência de um campo, além de evidenciar os pontos fortes e sugerir aperfeiçoamentos para tais teorias.
\end{abstract}

Palavras-chave dados abertos; teoria de campos de ação estratégica; transparência; governo aberto. 


\begin{abstract}
This article provides a reading of public policies for production and consumption of open data, according to the theory of strategic action fields. In Brazil, the government's open data field is characterized as emergent, since it has not been stabilized, yet. It is noticed that five groups engage in dynamics of cooperation and confrontation: a) State; b) internal governance units; c) civil society organizations; d) universities; and e) companies. The field dynamics are influenced by the possession of resources, or capitals, according to Pierre Bourdieu's terminology. The capitals identified are: a) technical; b) social; c) symbolic; d) economic; and e) informational. By means of a qualitative analysis of narratives reported by the subjects who participated in the research, the study presents an agenda for negotiation and action, involving the various participants in the field. It is worth noticing that these narratives are associated with transparency functions, which correspond to the open government concerns brought mainly by international legitimating agents. From the academic viewpoint, the research contributed to show a way to operationalize concepts proposed by theories devoted to strategic action fields, especially with regard to the state of emergency in a field, besides highlighting the strengths and suggest improvements for such theories.

Key words open data; theory of strategic action fields; transparency; open goverment.
\end{abstract}

\title{
Introdução
}

Este artigo retoma referências apresentadas por (Barbalho \& Medeiros, 2015). Vários países passaram por amadurecimento em suas estruturas de democracia e de tecnologia da informação. Nesses Estados, governos, sociedade civil organizada, empresas e entidades reguladoras, além da academia, discutem, elaboram e atuam no sentido de oferecer alternativas que viabilizem uma maior participação dos cidadãos e o incremento da confiança no governo a partir de mais transparência (Janssen, 2011; Kassen, 2013). As ações desses grupos apontam a necessidade de que os mecanismos tecnológicos em torno da internet sejam os mediadores das principais práticas de transparência de governo. É dessa conjunção sociotécnica que surge o conceito de dados abertos, caracterizado pela livre disponibilidade de dados que podem ser usados irrestritamente por qualquer interessado (Bazilian et al., 2012).

Este artigo mostra a evolução das políticas públicas sobre dados abertos no Brasil, a partir de sua caracterização como campo de ação estratégica emergente. Também se objetiva identificar: a) como as funções de transparência contribuíram para formar o atual estado do campo; b) como o campo de dados abertos se relacionou com o de governo aberto; e c) quais foram os capitais necessários para as disputas e colaborações que estão em pauta no campo. 
Além desta introdução, o artigo apresenta outras quatro seções: a) revisão da literatura, que trata, sobretudo, dos conceitos de campos de ação estratégica e transparência; b) apresentação da metodologia utilizada na pesquisa; c) apresentação e discussão dos resultados; e d) conclusões.

\section{Revisão da literatura}

Segundo Fligstein e McAdam (2012, p. 3, tradução nossa), a noção de teoria de campos de ação estratégica está associada ao entendimento da existência de "ordens sociais de nível meso como bloco estrutural básico da vida política e organizacional na economia, na sociedade civil e no Estado". Ainda de acordo com esses autores, campos de ação estratégica

[...] são ordens sociais de nível meso onde atores individuais ou coletivos interagem com conhecimento uns aos outros, utilizando compreensões compartilhadas com respeito aos propósitos do campo, às relações dentro do campo, a incluir quem possui poder e porque o possui, e às regras a observar (Fligstein \& McAdam, 2011, p. 3, tradução nossa).

Como propõem Fligstein e McAdam (2012), são três os estados em que um campo de ação pode se encontrar, quais sejam: a) emergência; b) estabilidade; e c) crise, a depender da maturidade dos entendimentos compartilhados pelos sujeitos sobre o que está em questão no campo, os relacionamentos dentro deste e as regras que o governam. O primeiro estado é o que se enfoca neste estudo, definido como "uma arena ocupada por dois ou mais atores cujas ações são orientadas um pelo outro, mas onde acordos sobre as condições básicas do campo ainda não emergiram" (Fligstein \& McAdam, 2011, p. 11, tradução nossa).

Dos três estados, a emergência é o mais desafiador para pesquisas sistematizadas, em função, principalmente, da ausência de significados amplamente compartilhados (Fligstein \& McAdam, 2012). Ainda assim, a possibilidade de se estudar um campo de ação estratégica em seus momentos de formação traria contribuições para a compreensão de como as características presentes nos momentos iniciais auxiliam na formação dos significados e das regras compartilhados do campo de ação que eventualmente surja. Destacam-se, entre essas características: a) os potenciais arranjos de poder analisados a partir das disparidades de recursos dos atores envolvidos; b) as possíveis formas de participação que se apoiarão nas habilidades sociais dos atores pioneiros; c) a extensão do papel do Estado como ator colaborador ou restritor da estabilidade do campo; e d) o apoio das unidades internas de 
governança (UIGs) na elaboração de rotinas e dos termos para a estabilidade do campo (Fligstein \& McAdam, 2012).

Por ser uma abordagem recente, grande parte dos países ainda está experimentando seus primeiros passos na definição de uma política pública associada à legitimação das formas de atuação dos atores que produzem e consomem dados abertos governamentais. Observa-se, a partir da leitura de trabalhos como os de Craveiro, Santana e Albuquerque (2013), Jaeger e Bertot (2010), Janssen (2011), Janssen e Estevez (2013), Kassen (2013) e Kierkegaard (2009), que já se notam iniciativas tomadas por parte dos governos que reconhecem organizações da sociedade civil como partes interessadas e atuantes na dinâmica de transparência governamental, capazes, inclusive, de oferecer ressignificados aos dados que são abertos e influenciar políticas públicas as mais diversas.

Confrontando as características desses achados da literatura com o que já foi apresentado sobre campos de ação estratégica emergente, tem-se que as iniciativas observadas são caracterizadas pela existência de atores estratégicos; no caso, governos e organizações da sociedade civil, que levam em consideração uns aos outros em suas ações. Além disso, observa-se, mormente nos textos de Craveiro et al. (2013) e Janssen (2011), que o espaço social onde essa ação ocorre ainda é dominado por ambiguidades e indefinições associadas: a) a conceito e princípios de dados abertos; b) ao papel do Estado sobre a produção e/ou consumo dos dados; c) ao marco regulatório para reúso de dados governamentais; e d) a modelos de negócio para garantir a sustentabilidade, no longo prazo, de aplicações que trabalhem com dados abertos.

Para este artigo, deve-se destacar três aspectos importantes para a emergência de um campo de ação estratégica. O primeiro é o entendimento do papel dos recursos, conceito discutido aqui sob a ótica dos capitais, a partir das contribuições de Pierre Bourdieu. $O$ segundo aspecto é a participação do Estado e o terceiro é a criação das chamadas UIGs. Em relação ao primeiro aspecto, a capacidade de um participante de um campo legitimar as práticas ou transformar o que está posto nas regras do jogo é diretamente relacionada à sua posse de um estoque de capital que interesse ao campo em que atua (Bourdieu \& Wacquant, 1992; Brandão, 2010).

Em relação à participação do Estado, tem-se que este é comumente envolvido na formação de um acordo para estabilidade de um campo e na criação de outros novos. Tal envolvimento é decorrente de seu potencial de estar presente em grande parte das relações entre grupos sociais. Por um lado, faz com que o Estado possa trabalhar na legitimação do campo em emergência, dado seu papel de estabelecimento de regras e normas para os atores sociais, ou seja, o de monopolista da violência e o de interventor financeiro e jurídico nas relações entre os outros atores (Bourdieu, 2008; Fligstein \& McAdam, 2012). Por outro lado, de forma cíclica, ao desempenhar esses papéis, o Estado acaba por gerar um conjunto de novos campos estatais e não estatais que têm a incumbência de dar estabilidade às 
novas dinâmicas advindas da expansão de regras e novas arenas sociais (Fligstein \& McAdam, 2012). É na definição de agenda de ação que se propõem os parâmetros de políticas públicas, entendidas como as alternativas e ideias práticas que tendem a ser implementadas pelo governo (Kingdon, 1995). Para Fligstein e McAdam (2012), nessa definição são usadas táticas de habilidades sociais caracterizadas, principalmente, por negociações em que se busca convencer grupos e pessoas de que uma agenda particular é do interesse desses atores sociais aos quais se tenta aliciar.

O terceiro aspecto importante para a emergência de um campo, a criação de UIGs, está associado à necessidade de assegurar que as regras do campo estejam sendo observadas dentro do que se espera de uma conformidade. Apesar de apresentarem-se quase sempre como atores neutros que são criados com o já referido propósito de velar pela observação de padrões e regras apontadas como legítimas em um campo consolidado ou em formação, a percepção é de que tais atores atuam, na prática, como linha auxiliar dos incumbentes dentro de um campo (Fligstein \& McAdam, 2012).

Para Fligstein e McAdam (2012), são três as formas como as UIGs contribuem com os incumbentes. A primeira é que, ao fazer o trabalho de governança, liberam os incumbentes para se dedicar mais às suas estratégias associadas à gestão do campo e de liderança (Phillips, 2012). A segunda é que a criação das UIGs contribui para tornar mais naturais as lógicas e regras do campo que tendem a atender com mais frequência aos interesses dos incumbentes. Esse tipo de condução pode ser visto, por exemplo, nos esforços de organizações que tratam de padronizações de tecnologia, que costumam levantar barreiras para mudanças de padrões que não atendam aos interesses diretos de multinacionais ocidentais, como relatam Lee e Oh (2006). Por fim, as UIGs tendem a atuar como ligação entre o campo de ação estratégica e outros campos externos importantes, já que esses atores costumam ter presença em mais de um campo.

Vale observar que a discussão das UIGs por Fligstein e McAdam (2012) privilegia os estados de campos estratégicos nos quais já estão definidos incumbentes e insurgentes e as regras já estão definidas, a ponto de ser útil uma instituição que assuma o papel de fiscalizador. Esse não é o caso do estado de emergência. A atuação de UIGs no estado de emergência, a forma com que se realiza essa atuação e mesmo o surgimento desses atores já nesse estado são questões em aberto que esta pesquisa pode ajudar a responder.

Para este artigo, as discussões estão centradas sobre as políticas públicas de dados abertos como um campo de ação estratégica emergente; dessa forma, faz-se necessária uma discussão sobre conceitos importantes que influenciam as dinâmicas do campo. As características de práticas de transparência na era eletrônica apontam que as tecnologias de informação passam a ser legitimadas como uma ferramenta primordial para a rápida disseminação e ampliação de alcance do que precisa ser, de alguma forma, submetido à transparência (Brandau, Endenich, Trapp, \& Hoffjan, 2012; Henderson, Sheetz, \& Trinkle, 
2012; Kim, Kim, \& Lee, 2009; Lodhia, 2012; Saxton, 2012; Unerman \& Bennett, 2004). No entanto, a transparência não pode ser um fim em si (Burger \& Owens, 2010; Nielsen \& Madsen, 2009; Stiglitz, 1999). Neste estudo se levantam alguns propósitos ou funções da transparência, discutidos a seguir.

Kierkegaard (2009) destaca o surgimento da chamada e-democracia. Essa abordagem pode ser entendida como o uso de tecnologias de comunicação eletrônica para aumentar o processo democrático. A e-democracia permite uma vasta oportunidade de ampliar a participação cidadã, além de proporcionar aos governos uma possibilidade dos cidadãos confiarem em suas ações (Kierkegaard, 2009). Encontram-se, então, duas funções da transparência para o setor público: a) confiança no governo e nas instituições; e b) participação cidadã. Outras funções trazidas da revisão da literatura são: a) planejamento estratégico; b) direcionamento de produtos e serviços; e c) exercício da cidadania.

\section{Metodologia}

Este trabalho utiliza a estratégia de estudo de caso aprofundado. Para Godoy (1995), o propósito fundamental de tal estratégia é estudar uma unidade social, independentemente se a referência for um indivíduo, ou um grupo. No caso deste artigo, a unidade social investigada é o espaço social formado em torno das práticas de produção e consumo de dados abertos.

Segundo Benbasat, Goldstein e Mead (1987), o estudo de caso é indicado quando a teoria e a prática sobre determinado assunto ainda estão em seu estágio inicial. Isso é observado já que a teoria de campos de ação estratégica foi formulada há poucos anos, em Fligstein e McAdam (2012). Benbasat et al. (1987) indicam que esse tipo de estratégia de pesquisa é adequada para situações em que são importantes a experiência e perspectiva dos atores e o contexto da ação é considerado crítico. Argumenta-se que o campo ainda em formação tem em seus participantes as principais fontes para o entendimento do que pode ser construído e da expectativa de como vai se organizar o campo, caso eventualmente se estabeleça, tendo como ponto de partida o contexto de um campo ainda em emergência.

Parte dos dados da pesquisa de campo foi coletada a partir de entrevistas semiestruturadas. Tais coletas foram feitas entre outubro e dezembro de 2013. Envolveram pessoas que representavam atores coletivos nas interações ao redor de formação de política pública sobre produção e consumo de dados abertos governamentais. Os tipos de sujeitos representados nas entrevistas são UIG, governo federal, sociedade civil, empresa pública, empresa privada e universidade. Uma pesquisa bibliográfica que antecedeu à pesquisa de campo apontou esses tipos de atores como possíveis participantes de um campo emergente com base tecnológica. 
Além de entrevistas, foram utilizados documentos para as análises qualitativas. Destacam-se: extrato do guia de orientação técnica sobre dados abertos produzido pelo World Wide Web Consortium (W3C); proposta de pesquisa sobre políticas públicas de dados abertos produzido pela Comissão Econômica para América Latina e Caribe (Cepal) e pelo W3C; edital de concurso de software produzido pelo W3C e pela Cepal; instrução normativa (IN) que institucionaliza a Infraestrutura Nacional de Dados Abertos (Inda); plano de ação da Inda; Lei de Acesso à Informação (LAI); relatório da primeira reunião do Grupo de Trabalho (GT) Dados Abertos organizado pelo W3C; plano de negócios do Open Data Institute (ODI); descritivo da ferramenta Plataforma IOTA, desenvolvida pela Aware Tecnologia em parceria com a Rede Nossa São Paulo; relatório da Open Government Partnership (OGP); descritivo do site De Olho nos Planos, desenvolvido pela Ação Educativa; relatório do Café Hacker, organizado junto à Secretaria Municipal de Saúde de São Paulo; devolutiva do Café Hacker, organizado junto à Secretaria Municipal de Transporte de São Paulo; e diretrizes do Fórum Econômico Mundial para dados abertos governamentais. Já os documentos considerados dados secundários são nove artigos publicados em um blog produzido pelo W3C.

Relata-se, ainda, que ocorreu coleta de dados em observação de eventos, no caso, dois seminários que trataram de alguma forma de dados abertos. O primeiro foi o São Paulo Aberta, que discutiu principalmente governo aberto, mas que teve pautas também associadas também a dados abertos. O segundo evento observado foi o Segundo Encontro Nacional de Dados Abertos. Para Miles, Huberman e Saldaña (2014), ao adotar essa abordagem de observação o pesquisador captura a percepção de indivíduos que não são necessariamente peças-chave. Nesse sentido, a observação de eventos, além de ter complementado as entrevistas que enfocaram os atores mais significativos, ofereceu a possibilidade de observar questões importantes para o quadro analítico deste estudo, principalmente as associadas aos efeitos das habilidades sociais e capitais dos atores pioneiros sobre os retardatários no campo.

Os dados foram analisados a partir da técnica de análise de conteúdo categorial (Bardin, 2009). Para essa atividade se utilizou a ferramenta At/as.TI como suporte para a codificação e visualização dos achados.

\section{Análise e discussão dos dados}

Nesta seção se apresentam os achados e as análises referentes à pesquisa de campo. Expõem-se os dados e análises associados às questões emergentes do campo de dados abertos governamentais e suas associações com as funções de transparência e os capitais necessários envolvidos no campo. Também há discussões sobre os processos de ressignificação dos dados pelos diversos atores e as relações do campo de dados 
abertos com outros campos, principalmente o de governo aberto. Para manter anônimos os entrevistados, as referências a eles seguem a codificação indicada no Quadro 1.

Quadro 1 - Codificação dos entrevistados

\begin{tabular}{|c|c|}
\hline Código & Descrição \\
\hline U1E1 & Entrevistado 1 da UIG 1 \\
\hline U1E2 & Entrevistado 2 da UIG 1 \\
\hline U2E1 & Entrevistado 1 da UIG 2 \\
\hline U2E2 & Entrevistado 2 da UIG 2 \\
\hline S1E1 & Entrevistado 1 da organização da sociedade civil 1 \\
\hline EP1E1 & Entrevistado 1 da empresa privada 1 \\
\hline EE1E1 & Entrevistado 1 da empresa estatal 1 \\
\hline O1E1 & Entrevistado 1 do órgão de governo 1 \\
\hline
\end{tabular}

Fonte: Elaborada pelo autor.

Uma primeira análise a ser destacada neste artigo é a exposta na Tabela 1, que aprofunda o olhar sobre as ações dos atores e as questões que se associam a tais ações, tendo como referência as funções da transparência. Essa tabela mostra em ordem decrescente de frequência algumas das 46 questões que surgem quando se analisam as ações dos atores que estão envolvidos na fase de formação de um acordo para o campo. Os valores que aparecem nas células se relacionam ao número de citações de cada uma das questões para cada uma das funções da transparência. As linhas selecionadas dizem respeito às 10 questões mais citadas na pesquisa de campo. 
Tabela 1 - Distribuição de pontos de questões emergentes entre as funções de transparência

\begin{tabular}{|c|c|c|c|c|c|c|}
\hline & $\begin{array}{c}\text { Direcionamento } \\
\text { de produtos } \\
\text { e serviços }\end{array}$ & Participação & $\begin{array}{l}\text { Exercício da } \\
\text { cidadania }\end{array}$ & Confiança & $\begin{array}{c}\text { Planejamento } \\
\text { estratégico }\end{array}$ & Totais \\
\hline $\begin{array}{l}\text { Viabilização } \\
\text { de modelo de } \\
\text { negócios para } \\
\text { dados abertos }\end{array}$ & 38 & 17 & 12 & 5 & 6 & 78 \\
\hline $\begin{array}{l}\text { Governo } \\
\text { eletrônico }\end{array}$ & 13 & 11 & 6 & 5 & 2 & 37 \\
\hline $\begin{array}{c}\text { Participação } \\
\text { social na } \\
\text { construção } \\
\text { de aplicativos } \\
\text { sobre dados } \\
\text { abertos }\end{array}$ & 13 & 15 & 6 & 3 & 0 & 37 \\
\hline $\begin{array}{c}\text { Dados } \\
\text { abertos como } \\
\text { recurso para } \\
\text { informação } \\
\text { inteligente }\end{array}$ & 12 & 8 & 7 & 2 & 7 & 36 \\
\hline $\begin{array}{l}\text { Envolvimento } \\
\text { de } \\
\text { organizações } \\
\text { do terceiro } \\
\text { setor no reúso } \\
\text { de dados } \\
\text { abertos }\end{array}$ & 12 & 9 & 6 & 2 & 3 & 32 \\
\hline $\begin{array}{c}\text { Hábito de } \\
\text { abertura de } \\
\text { dados }\end{array}$ & 12 & 12 & 4 & 1 & 2 & 31 \\
\hline
\end{tabular}




\begin{tabular}{|c|c|c|c|c|c|c|}
\hline $\begin{array}{c}\text { Explorar dados } \\
\text { abertos com } \\
\text { quem realmente } \\
\text { precisa }\end{array}$ & 8 & 10 & 5 & 4 & 2 & 29 \\
\hline $\begin{array}{l}\text { Estruturação de } \\
\text { rede para projetos } \\
\text { em dados abertos }\end{array}$ & 11 & 10 & 4 & 2 & 1 & 28 \\
\hline $\begin{array}{c}\text { Dados abertos } \\
\text { em processos de } \\
\text { desenvolvimento } \\
\text { de software }\end{array}$ & 11 & 6 & 5 & 2 & 1 & 25 \\
\hline $\begin{array}{l}\text { Qualidade dos } \\
\text { dados abertos }\end{array}$ & 10 & 8 & 4 & 2 & 1 & 25 \\
\hline
\end{tabular}

Fonte: Elaborada pelo autor.

De acordo com Fligstein e McAdam (2012), os atores são envolvidos em um campo emergente por outros com habilidades sociais, a partir de narrativas que lhes tragam significados e noções de pertencimento. As questões listadas na Tabela 1 estão associadas a esses tipos de narrativas. Entende-se que um eventual acordo será pautado em grande medida por tais questões, já que elas trazem essa noção de significados e pertencimento que são determinantes para a identificação dos limites do campo e sua rotinização. Observa-se que nas narrativas da Tabela 1 constam questões associadas à governança trazidas principalmente pelas UIGs, a distribuição dos interesses do terceiro setor entre as cinco funções de transparência, e a concentração do interesse do setor produtivo sobre o desenvolvimento de produtos e serviços.

No topo da Tabela 1 se encontra a viabilização de modelo de negócios para dados abertos. Há o entendimento de que a sustentabilidade do campo está relacionada com a construção de um modelo de negócio atrativo, principalmente para empresas privadas e organizações não governamentais (ONGs), que permita um contínuo fluxo de abertura e consumo de dados governamentais. O modelo de negócio está associado, principalmente, à construção e disponibilização de produtos e serviços, já que essa é a forma como as ressignificações sobre os dados abertos são instrumentalizadas e, por consequência, é como as empresas e ONGs podem manifestar-se em torno do uso de dados abertos. Ao 
mesmo tempo, esses produtos e serviços visam a atender às necessidades de participação cidadã, exercício da cidadania, confiança no governo e planejamento estratégico. Dessa forma, todas as funções da transparência têm relações de causa e efeito com a viabilização de um modelo de negócios para o campo. Parece provável, ainda, que a viabilização do modelo de negócio guarda relações com várias outras questões listadas na Tabela 1.

Entende-se que a teia de relacionamentos que surge quando se busca viabilizar um modelo de negócio para o campo e as demais questões que emergem na agenda de estabilização do campo é, em grande medida, influenciada pelos capitais disponíveis pelos diversos atores. A Tabela 2 mostra a influência dos diversos capitais nas dez questões emergentes com maior média de citação.

Tabela 2 - Distribuição do uso dos capitais nas questões emergentes

\begin{tabular}{|c|c|c|c|c|c|c|}
\hline Questões emergentes & $\begin{array}{l}\text { Capital } \\
\text { técnico }\end{array}$ & $\begin{array}{c}\text { Capital } \\
\text { informacional }\end{array}$ & $\begin{array}{l}\text { Capital } \\
\text { simbólico }\end{array}$ & $\begin{array}{l}\text { Capital } \\
\text { social }\end{array}$ & $\begin{array}{c}\text { Capital } \\
\text { econômico }\end{array}$ & Média \\
\hline $\begin{array}{l}\text { Viabilização de modelo de } \\
\text { negócios para dados abertos }\end{array}$ & 0,37 & 0,18 & 0,07 & 0,29 & 0,20 & 0,22 \\
\hline Governo eletrônico & 0,13 & 0,31 & 0,20 & 0,12 & 0,00 & 0,15 \\
\hline $\begin{array}{l}\text { Estruturação de rede para } \\
\text { projetos em dados abertos }\end{array}$ & 0,13 & 0,14 & 0,11 & 0,15 & 0,14 & 0,13 \\
\hline $\begin{array}{l}\text { Hábito de abertura de } \\
\text { dados }\end{array}$ & 0,10 & 0,13 & 0,27 & 0,07 & 0,03 & 0,12 \\
\hline $\begin{array}{c}\text { Envolvimento de } \\
\text { organizações do terceiro } \\
\text { setor no reúso de dados } \\
\text { abertos }\end{array}$ & 0,17 & 0,02 & 0,03 & 0,19 & 0,14 & 0,11 \\
\hline $\begin{array}{c}\text { Explorar dados abertos com } \\
\text { quem realmente precisa }\end{array}$ & 0,16 & 0,12 & 0,08 & 0,12 & 0,04 & 0,10 \\
\hline $\begin{array}{l}\text { Municípios como pontos } \\
\text { focais de dados abertos }\end{array}$ & 0,08 & 0,20 & 0,04 & 0,05 & 0,13 & 0,10 \\
\hline
\end{tabular}




\begin{tabular}{|c|c|c|c|c|c|c|}
\hline Qualidade dos dados abertos & 0,12 & 0,15 & 0,14 & 0,08 & 0,00 & 0,10 \\
\hline $\begin{array}{c}\text { Dados abertos como recurso } \\
\text { para informação inteligente }\end{array}$ & 0,10 & 0,13 & 0,08 & 0,06 & 0,09 & 0,09 \\
\hline $\begin{array}{c}\text { Envolvimento de empresas } \\
\text { no processo de consumo de } \\
\text { dados abertos }\end{array}$ & 0,05 & 0,14 & 0,07 & 0,06 & 0,10 & 0,08 \\
\hline
\end{tabular}

Fonte: Elaborada pelo autor.

Pelos valores da Tabela 2, que mostra as proporções de citações de cada célula em relação ao total de citações, evidencia-se que os capitais social e técnico são predominantes nas questões que dominam a agenda de formação de acordo para o campo, embora os capitais simbólico, informacional e econômico também sejam importantes para algumas questões específicas.

Os capitais técnico e social compartilham os mesmos conjuntos de atores e têm influências aproximadas nas questões emergentes. Nos dois, destacam-se entre as manifestações desses capitais as questões associadas à viabilização de um modelo de negócio, estabelecimento de redes e envolvimento de organizações da sociedade civil. Isso é comprovado nos dados da pesquisa de campo em projetos como os que atores do terceiro setor, como a Fundação Avina, Ação Educativa, Rede Nossa São Paulo, entre outros, articulam-se com organizações de hackers ativistas ou mesmo com empresas privadas. Observa-se, nesses casos, complementaridade de capitais técnicos, que é viabilizada por meio das articulações em redes e alianças, que são, por sua vez, manifestações dos capitais sociais desses atores.

A pesquisa de campo sugere que as iniciativas de desenvolvimento de soluções pontuais em que se articulem os capitais técnico e social não são suficientes para que o campo adquira sua estabilidade. Haveria, ainda, a necessidade de um arcabouço de governança que proporcione previsibilidade legal ao campo, rotinize a abertura de dados por parte dos órgãos de governo e viabilize a sustentabilidade de projetos que requerem, muitas vezes, o envolvimento da iniciativa privada. Essa governança está associada ao capital simbólico, posto que diz respeito à legitimidade dos atores em relação às suas ações para disciplinamento do campo. Já a rotinização de abertura de dados requererá redistribuição do capital informacional dos que possuem os dados a ser abertos. Por sua vez, a sustentabilidade de projetos implica a atração ou o desenvolvimento de capitais econômicos. 
Observa-se que o capital simbólico associado à governança é manifestado, principalmente, pelos atores que se movem em torno do governo eletrônico e das questões que têm relações mais fortes com esse tema, tais como o hábito de abertura de dados, a participação social na política de dados abertos, a legitimidade da cobrança por dados abertos e a qualidade dos dados abertos. Dos dados da pesquisa de campo, constata-se que o capital simbólico presente nesse conjunto de temáticas é exercido principalmente por UIGs e agentes de governo. A legitimidade de cobrança das UIGs se sustenta em dois tipos de fonte: a) a coercitiva, que é o caso da Secretaria de Logística e Tecnologia da Informação (SLTI), da Controladoria-Geral da União (CGU) e do Tribunal de Contas da União (TCU), cujas atribuições são previstas por instrumentos legais; e b) a normativa, que é o caso do W3C e OGP, cujas influências são reconhecidas pelo conjunto de atores que compõem o campo, mesmo sem a necessidade de instrumentos legais.

Infere-se que caberia às UIGs utilizar seus capitais simbólicos para definir os padrões a ser observados na abertura de dados, articular legislações específicas sobre o tema e divulgar melhores práticas observadas. Já aos agentes de governo caberia fazer cumprir em suas estruturas burocráticas as práticas recomendadas pelas UIGs e eventualmente observadas em órgãos de governo referenciais, por meio de mecanismos coercitivos ou miméticos.

Um dos principais desafios à construção da legitimidade por parte das UIGs e, principalmente, dos órgãos de governo é convencer os demais participantes do campo de que há, de fato, interesse dos detentores do capital simbólico, que no caso dos órgãos governamentais também são detentores do capital informacional na construção de um campo sustentável. Os dados revelam desconfianças e receios de que os interesses expressos por alguns atores a favor da transparência sejam meras respostas pontuais a demandas também pontuais. Isso está presente nas evidências associadas às questões "Resposta às Jornadas de Junho"1 e "Interesse do Estado Limitado à Legitimação".

Em relação à primeira questão, S1E1 indica que, apesar de bem-vindas, há dúvidas sobre a sustentabilidade de várias iniciativas de governo eletrônico e transparência que ocorreram logo após as Jornadas de Junho de 2013 - dado o envolvimento ainda pontual dos atores de governo, há a possibilidade de que esse interesse renovado expresso seja uma mera reação aos movimentos da sociedade que ocorreram, também, de forma pontual e pouco organizada (informação verbal)2 . Já em relação à segunda questão, U2E1 entende que muitas das ações de governo, principalmente os hackathons são eventos pontuais, que apesar de importantes, não chegam a desenvolver uma cultura, tendo um papel mais importante do ponto de vista de indicar para a sociedade, principalmente para os hackers

1 “Jornadas de Junho" é uma referência aos protestos e às manifestações que ocorreram em várias cidades brasileiras em junho de 2013. Mais informações sobre o que caracterizou essas manifestações podem ser encontradas em Rolnik (2013). 2 Conforme entrevista com S1E1. 
ativistas, que o governo tem ações concretas para abertura de dados (informação verbal) ${ }^{3}$. Para U2E1, um efeito desses eventos é que os envolvidos nos hackathons acabam por legitimar ações que são pontuais, mas que deveriam ser parte de uma política sistematizada e disseminada no setor público (informação verbal) ${ }^{4}$.

O capital econômico tem um potencial de relevância identificado, principalmente, ao redor da questão com mais referências do levantamento feito neste estudo, a viabilização de um modelo de negócio. Há várias evidências de que é possível desenvolver projetos em que haja retornos econômicos, permitindo, assim, a atratividade maior de atores de capital privado que poderiam trazer aportes ao campo. Como indicado anteriormente, entende-se que a sustentabilidade do campo passa pelas oportunidades de atrair esse tipo de capital, seja para apoiar projetos de ONGs, tal como já conseguem fazer OKFN (Open Knowledge Foundation) e ODI, seja para remunerar empresas privadas, principalmente startups. As evidências empíricas da pesquisa sugerem que aquilo que as empresas esperam do governo para se engajar com maior afinco é, principalmente, a rotinização da publicação de dados abertos, para que haja a possibilidade de consolidar modelos de negócio associados à previsibilidade de alimentação dos dados requeridos pelos projetos que utilizam os dados abertos pelos órgãos governamentais. Nesse sentido, suporta-se o entendimento de que o capital econômico visto como necessário para sustentabilidade do campo requer como pressuposto a disposição de diminuir a concentração do capital informacional por parte do Estado.

Em relação ao capital informacional, entende-se que, desde o início do movimento ao redor da questão de dados abertos até agora, esse capital está quase monopolizado pelos agentes de governo, em um nível micro, representados pelos gestores públicos, e em um nível meso, representados pelos órgãos da administração direta e indireta, e que a real possibilidade do campo vir a se estabelecer depende desses atores abrirem mão de seu monopólio. Algumas evidências da pesquisa de campo demonstram a força desse capital, como as entrevistas de S1E1 e EP1E1, que afirmam que no momento atual o que mais importa é que o dado tem de ser publicado, nem importa como, sendo esta a principal função do Estado (informação verbal) ${ }^{5}$. Nesse sentido, os propósitos do campo associados à transparência pressionam para que o poder associado à concentração do capital informacional seja relativizado. Atores que têm o capital simbólico, tais como a CGU e a SLTI, tendem a agir no sentido de forçar a disponibilização das informações. Não é à toa que os movimentos dos atores que buscam estabelecer acordos para a estabilização do campo procuram sempre dar ênfase às ações que busquem a legitimidade da cobrança por dados abertos. Além disso, a ênfase em explorar dados abertos com quem realmente

3 Conforme entrevista com U2E1.

4 Conforme entrevista com U2E1.

5 Conforme entrevista com S1E1 e EP1E1. 
precisa, como mostrado na Tabela 2, implica a desmistificação da informação produzida pela burocracia, que geralmente é de difícil compreensão por envolver um vocabulário e um racional hermético. Daí o surgimento de narrativas associadas a questões como o racional aberto, ou seja, demandas para uma explicação aprofundada das regras de negócio associadas aos dados. Nesse sentido, um dos eventos pesquisados, São Paulo Aberta, mostra como uma abordagem de hackathons pode ir além da simples abertura de dados para também explicar conceitos e harmonizar entendimentos sobre os significados para a administração pública dos dados disponibilizados.

Infere-se, portanto, que na medida em que esse tipo de ação se torne corriqueiro e o campo se torne estável e sustentável, haverá necessariamente uma perda do monopólio do capital informacional por parte dos órgãos do governo. Parece provável que as principais dinâmicas de mudança e permanência do campo se formarão ao redor da disputa desse capital e que se os detentores do capital informacional não perceberem ganhos com o esvaziamento deste, eles tenderão a buscar influenciar a governança do campo de modo que suas perdas sejam minimizadas. Pode-se imaginar uma situação em que alguns órgãos do governo pressionem para a abertura de dados enquanto outros tentem manter seu monopólio intacto. Em um extremo, as condições de mudança que as políticas de dados abertos poderiam apontar podem ser atenuadas pelos movimentos de permanência das regras do jogo executados pelos detentores do capital informacional. Por outro lado, as pressões por transparência vindas de ONGs, principalmente as internacionais, de gestores públicos que veem ganhos com as implementações e de UIGs que tenham o respaldo dos mais altos escalões da administração pública podem impor condições de mudança que garantam a sustentabilidade no longo prazo do campo, por meio da quebra do monopólio do capital informacional.

Uma discussão importante sobre o campo de dados abertos consiste nos fatores que levam o campo ao encontro de outros próximos, principalmente o de governo aberto. Essa discussão passa, principalmente, pelas ressignificações que os diversos atores fazem dos dados que são abertos pelo governo. Algumas das ressignificações observadas no processo de emergência do campo de dados governamentais abertos estão associadas às possibilidades presentes na própria natureza dos dados abertos, quais sejam, permitir que agentes que não produziram os dados possam utilizá-los para seus próprios propósitos. Identificou-se que, no Brasil, a política pública de dados abertos foca-se na dinâmica de agentes acessarem os dados disponibilizados, processarem-nos em aplicativos, buscarem visualizações alternativas dos dados e disponibilizarem essas novas visualizações por meio, principalmente, de recursos baseados na infraestrutura de internet.

A identificação dessas ressignificações mostra que o campo em emergência está, de alguma forma, cumprindo seus propósitos, principalmente o de participação social e o de desenvolvimento de novos produtos e serviços, a partir das interações entre os atores. 
Grande parte dos aplicativos produzidos que mostram as ressignificações decorre de concursos como hackathons, onde agentes de Estado disponibilizam os dados e incentivam outros atores a participar dos eventos. A participação dos outros agentes envolve não só a construção dos aplicativos que vão ressignificar os dados disponibilizados, mas também o questionamento do propósito inicial dos dados e da dinâmica que os geraram. Essas interações permitem que as partes envolvidas passem a ter melhor conhecimento sobre detalhes dos processos organizacionais e de políticas públicas que levaram à geração dos dados primários disponibilizados. Além disso, melhoram os aplicativos que serão desenvolvidos, aumentando a possibilidade de que as ressignificações tenham utilidade e sejam menos propícias a questionamentos por inconsistências de análise.

A leitura proporcionada pelos atores que ressignificam os dados apontam aos gestores públicos, ou formuladores de políticas públicas, possibilidades de entender os efeitos de seus processos de formulação e execução de ações a partir de uma visão sob uma ou mais óticas que representam conjuntos de atores diferenciados. Nesse sentido, um mesmo conjunto de dados, como, por exemplo, o do Instituto Nacional de Estudos e Pesquisas Educacionais (Inep), que foi utilizado em hackathons, pode ser ressignificado por diversos grupos de atores que representem diversas partes interessadas na educação pública, a saber: estudantes, pais de estudantes, diretores de escola, professores, fornecedores, organismos internacionais, partidos políticos, ONGs, entre outros. Além disso, esses mesmos atores, instrumentalizados por esses dados ressignificados, podem atuar com mais consistência na defesa de seus pontos de vistas por meio de mecanismos de participação que são oferecidos pelo Estado.

Do ponto de vista da contribuição para a teoria de campos de ação estratégica, os processos de ressignificação de dados abertos evidenciam um ponto relevante levantado por Fligstein e McAdam (2012), associado ao diálogo e à inter-relação entre campos estratégicos. De acordo com os achados da pesquisa, os campos que discutem a participação popular na gestão pública, como, por exemplo, o de governo aberto, é diretamente impactado pelas possibilidades trazidas pelas ressignificações dos dados disponibilizados. Da mesma forma, os campos associados às diversas políticas públicas que estejam sendo retratadas pelos dados abertos, como o da educação pública, no caso dos dados do Inep, também podem sofrer influência das interações dos atores do campo emergente de políticas públicas de dados abertos. As regras do jogo que estabelecem os limites dos campos que são impactados pelas ressignificações dos dados abertos não chegam necessariamente a ser questionadas, mas os atores que estão nesses campos são influenciados em suas ações pelos resultados das novas leituras dos dados. Em outras palavras, os parâmetros de regras sobre participação social no campo do governo aberto, por exemplo, não são influenciados pelos resultados alcançados pelas ações de ressignificação, até porque isso já estava nas regras do jogo; porém, os atores do campo de governo aberto passam a se sentir mais 
confiantes de que a sustentabilidade do campo de dados abertos trará contribuições para a defesa de suas posições. Da mesma forma, as regras de políticas públicas em educação não serão necessariamente abaladas com leituras sobre os dados do Inep. Não obstante, os atores que atuam nesse campo se utilizarão das ressignificações para defender suas posições ou buscar ameaçar a posição de outros atores estratégicos.

Em resumo, as ressignificações de dados são efeitos de interações entre os atores que atuam no campo emergente de políticas públicas de dados abertos. As evidências deste estudo sugerem que tais interações ocorrem ou ocorrerão principalmente: a) no chamamento à ressignificação de dados governamentais disponibilizados; b) na discussão e no aprimoramento dos dados disponibilizados; c) na compreensão das leituras dos dados já ressignificados; d) na participação social nos fóruns sobre políticas públicas associadas aos dados ressignificados.

As ressignificações de dados, por sua vez, trazem efeitos sobre a aposta na ressignificação do conceito de transparência que está por trás de iniciativas como a OGP. As iniciativas de governo aberto partem do pressuposto de que a transparência permite maior participação da sociedade nas decisões de governo, melhorando a eficácia governamental e tornando os cidadãos agentes ativos da democracia. Na medida em que as primeiras ressignificações de dados abertos começam a ganhar visibilidade, pode-se prever um reforço da legitimação do novo paradigma sobre transparência baseada no forte uso de tecnologia da informação e na adesão a práticas legitimadas. O formato das ressignificações de dados pode vir a fortalecer determinadas ideias em disputa sobre o conceito de dados abertos. As iniciativas mais relevantes penderão a balança para os projetos em disputa que mais se aproximam de sua implementação. Nesse sentido, estarão sendo dados passos importantes para a diminuição de ambiguidades e o fortalecimento da percepção de identidade entre atores que agem ou venham a agir no campo. Assim, o processo de ressignificação se mostra altamente relevante para o alcance de acordos ao redor de pontos em disputa e, consequentemente, para o efetivo estabelecimento do campo.

\section{Conclusões}

Este estudo demonstrou quais são os atores envolvidos no campo emergente de políticas públicas de dados abertos, tanto no Brasil como no mundo, o impacto das novas tecnologias, os caminhos já percorridos, os diversos capitais (recursos) relevantes para o campo e as possíveis influências sobre a consolidação ou não do campo e sua eventual conformação. Demonstrou-se, ainda, como a produção e o consumo de dados abertos se relacionam com as chamadas "funções da transparência". 
Esta pesquisa possibilitou a identificação de três grandes temas relacionados às interações entre os atores, quais sejam: a ressignificação dos dados abertos; a influência do ambiente normativo internacional, principalmente do campo de governo aberto representado pela OGP; e as dinâmicas associadas à posse e ao uso dos capitais observados no campo. Esses grandes temas foram analisados em conjunto para indicar o atual estado do campo e quais são as perspectivas para sua eventual consolidação.

O estudo contribui, ainda, com o levantamento - a partir de narrativas que tendem a dominar as pautas dos acordos que influenciarão a estabilização do campo - de uma extensa agenda para negociação e ação. Essa agenda pode ser do interesse das partes interessadas principalmente por oferecer uma visão abrangente baseada nas funções da transparência, no uso de capitais e nas possibilidades de atuação dos diversos atores envolvidos.

\section{Referências}

Bardin, L. (2009). Análise de conteúdo. Lisboa, Portugal: Ed. 70.

Barbalho, F. Medeiros, J. A emergência de políticas públicas de dados abertos governamentais: 0 caso brasileiro sob a ótica das funções da transparência e da teoria de campos de ação. $\ln X X$ Congreso Internacional del CLAD. Lima, 2015.

Bazilian, M., Rice, A., Rotich, J., Howells, M., DeCarolis, J., Macmillan, S., ... Liebreich, M. (2012). Open source software and crowdsourcing for energy analysis. Energy Policy, 49, 149-153.

Benbasat, I., Goldstein, D., \& Mead, M. (1987). The case research strategy in studies of information system. MISS Quartely, 11(3), 369-386.

Bourdieu, P. (2008). Razões práticas: sobre a teoria da ação. Campinas, SP: Papirus.

Bourdieu, P., \& Wacquant, L. (1992). An invitation to reflexive sociology. Chicago, IL: University of Chicago Press.

Brandão, Z. (2010). Operando com conceitos: com e para além de Bourdieu. Educação e Pesquisa, 36(1), 227-241.

Brandau, M., Endenich, C., Trapp, R., \& Hoffjan, A. (2012). Institutional drivers of conformity: evidence for management accounting from Brazil and Germany. International Business Review, 22(2), 466-479.

Burger, R., \& Owens, T. (2010). Promoting transparency in the NGO sector: examining the availability and reliability of self-reported data. World Development, 38(9), 1263-1277.

Craveiro, G., Santana, M., \& Albuquerque, J. (2013). Assessing open government budgetary data in Brazil. In Annals of the 7th International Conference on Digital Society. Nice, France.

Fligstein, N., \& McAdam, D. (2011). Toward a general theory of strategic action fields. Sociological Theory, 29(1), 1-26. 
Fligstein, N., \& McAdam, D. (2012). A theory of fields. Oxford, England: Oxford University Press.

Godoy, A. (1995). Pesquisa qualitativa: tipos fundamentais. Revista de Administração de Empresas, 35(3), 20-29.

Henderson, H., Sheetz, S., \& Trinkle, B. (2012). The determinants of inter-organizational and internal in-house adoption of XBRL: a structural equation model. International Journal of Accounting Information Systems, 13(2), 109-140.

Jaeger, P., \& Bertot, J. (2010). Transparency and technological change: ensuring equal and sustained public access to government information. Government Information Quarterly, 27(4), 371-376.

Janssen, K. (2011). The influence of the PSI directive on open government data: an overview of recent developments. Government Information Quarterly, 28(4), 446-456.

Janssen, M., \& Estevez, E. (2013). Lean government and platform-based governance: doing more with less. Government Information Quarterly, 30(Suppl. 1), 51-58.

Kassen, M. (2013). A promising phenomenon of open data: a case study of the Chicago open data project. Government Information Quarterly, 30(4), 508-513.

Kierkegaard, S. (2009). Open access to public documents: more secrecy, less transparency! Computer Law \& Security Review, 25(1), 3-27.

Kim, S., Kim, H., \& Lee, H. (2009). An institutional analysis of an e-government system for anti-corruption: the case of OPEN. Government Information Quarterly, 26(1), 42-50.

Kingdon, J. (1995). Agendas, alternatives, and public policies. New York, NY: HarperCollins College Publishers.

Lee, H., \& Oh, S. (2006). A standards war waged by a developing country: understanding international standard setting from the actor-network perspective. Journal of Strategic Information Systems, 15(3), 177-196.

Lodhia, S. (2012). Web based social and environmental communication in the Australian minerals industry: an application of media richness framework. Journal of Cleaner Production, 25, 73-85.

Miles, M., Huberman, A., \& Saldaña, J. (2014). Qualitative data analysis: a method sourcebook. Thousand Oaks, CA: Sage.

Nielsen, C., \& Madsen, M. (2009). Discourses of transparency in the intellectual capital reporting debate: moving from generic reporting models to management defined information. Critical Perspectives on Accounting, 20(7), 847-854.

Phillips, J. (2012). The seeds of power: "good governance” in Nigeria's debt-relief gains (Harvard Working Paper). Cambridge, MA: Harvard.

Rolnik, R. (2013). Apresentação: As vozes das ruas: as revoltas de junho e suas interpretações. In Boitempo Editorial \& Carta Maior (Orgs.), Cidades rebeldes: passe livre e as manifestações que tomaram as ruas do Brasil. São Paulo, SP: Boitempo. 
Saxton, G. (2012). New media and external accounting information: a critical review. Australian Accounting Review, 22(3), 286-302.

Stiglitz, J. (1999). On liberty, the right to know, and public discourse: the role of transparency in public life (Oxford Amnesty Lecture Series). Oxford England: Oxford University.

Unerman, J., \& Bennett, M. (2004). Increased stakeholder dialogue and the internet: towards greater corporate accountability or reinforcing capitalist hegemony? Accounting, Organisations and Society, 29(7), 685-707. 